\title{
Can Batch or Semi-Batch Processes Save Energy in Reverse-Osmosis Desalination?
}

\author{
Revised and re-submitted to
}

\author{
Desalination
}

August 22, 2016

Jay R. Werber, ${ }^{1}$ Akshay Deshmukh, ${ }^{1}$ and Menachem Elimelech ${ }^{*}$ 


\section{Abstract}

35 Energy savings in reverse osmosis (RO) are highly constrained by the design of conventional 36 processes, for which the minimum practical energy of desalination substantially exceeds the 37 thermodynamic minimum. Batch processes can theoretically approach the thermodynamic 38 minimum, suggesting the possibility for further energy savings. In this study, we aim to quantify 39 what energy reductions may be possible for batch-like processes when process inefficiencies

40 such as frictional losses and concentration polarization are included. We first introduce a 41 practical batch process that utilizes energy recovery devices and an unpressurized feed tank. We 42 also consider a less practical pressurized-tank scenario, as well as semi-batch (closed-circuit) RO. 43 We then derive analytical approximations and conduct numerical modeling to compare the 44 energy requirements of batch, semi-batch, and staged RO processes under realistic conditions. 45 Through this analysis, we find that practical batch-like processes and processes with increased 46 staging offer comparable and significant energy savings. For example, semi-batch RO and two47 stage RO would save $13 \%$ and $15 \%$ energy, respectively, over one-stage seawater RO at $50 \%$ 48 recovery. We conclude with a discussion of other important factors, such as capital costs and 49 process robustness and flexibility, that will affect the implementation of batch, semi-batch, and 50 staged processes.

\section{1}

52

53

54

\section{Keywords}

56 Batch reverse osmosis; semi-batch closed-circuit reverse osmosis; staged reverse osmosis;

57 energy of desalination; process design

\section{GRAPHICAL ABSTRACT}




\section{Introduction}

With the need to alleviate water scarcity in water-stressed regions around the world, desalination of saline waters such as seawater and brackish groundwater has become an increasingly important and widespread technology [1, 2]. Membrane-based desalination, particularly reverse osmosis (RO), makes up the vast majority of new installations, largely owing to its high energy efficiency and low operating costs relative to thermal desalination [3]. The cost and energy requirements for RO have decreased considerably over the last few decades. For example, in seawater RO (SWRO), the specific energy (i.e., the amount of energy required per volume of permeate) in the desalination stage has decreased from over $15 \mathrm{kWh} \mathrm{m}^{-3}$ in 1970 to around $2 \mathrm{kWh} \mathrm{m}^{-3}$ today [2]. This decrease in specific energy stems from the advent of highly permeable thin-film composite membranes, the increased efficiency of pumps, and the use of high-efficiency energy recovery devices (ERDs) to recover hydraulic energy from the highpressure brine $[2,3]$. Despite the substantial progress, energy remains an important consideration; energy usage can still be up to 50\% of a SWRO facility's operation and maintenance costs [3].

The energy consumption in brackish water RO (BWRO) is typically lower than SWRO and considerably more variable, as the total dissolved solids (TDS) content of brackish groundwater varies over a wide range (1000-10000 ppm) from site to site [3,4]. While energy consumption is a relatively low concern for low-salinity feed water, brackish feed streams with greater salinity can require substantial energy consumption, especially when operated at high recoveries. In addition, there is often a strong driver to maximize recovery due to concerns over disposal of the high concentration brine, especially for inland locations [3,4]. Despite this driver, achieving high recoveries is often technically challenging due to the sharp increase in concentration factor (i.e., the brine solute concentration divided by the feed concentration) at very high recoveries, which can lead to scaling and sharply increased osmotic pressure. Hence, there is a need to develop processes that can achieve very high recoveries in a robust and efficient manner.

$84 \mathrm{RO}$ is also increasingly applied for the treatment of municipal and industrial wastewaters [5]. Salinities are relatively low in municipal wastewater, typically less than 1,000 ppm TDS [6], and require relatively low energy for RO treatment. In contrast, industrial wastewaters, such as

87 wastewaters from the chemical, pharmaceutical, and power industries, can vary much more 
broadly in solution composition and can have more stringent treatment goals. In the most extreme example, some plants must operate zero liquid discharge (ZLD) schemes because of waste disposal concerns [7, 8]. ZLD processes invariably consume large amounts of energy due to the need to separate all of the water from solids. As the most efficient desalination technology available, RO will play a large role in ZLD schemes and will need to be operated at very high recoveries as efficiently as possible.

Owing to the ever-increasing desalination capacity of RO facilities, further improvements in energy consumption would have considerable impact. Energy savings from further membrane advances will be rather limited, as increased membrane water permeability above currently achieved levels (2-3 $\mathrm{L} \mathrm{m}^{-2} \mathrm{~h}^{-1}$ bar $^{-1}$ for SWRO) would have only a minor impact [9]. Recent modeling studies indicate that even a large increase in the membrane water permeability coefficient from 2 to $10 \mathrm{~L} \mathrm{~m}^{-2} \mathrm{~h}^{-1}$ bar $^{-1}$ would yield at best a $4 \%$ decrease in energy consumption for SWRO [9]. This limited decrease is partly due to the energetics being highly constrained by conventional, one-stage operation in SWRO [10]. Current energy requirements $\left(\sim 2 \mathrm{kWh} \mathrm{m}^{-3}\right)$ already approach the practical minimum specific energy for conventional, one-stage SWRO, which is approximately $1.6 \mathrm{kWh} \mathrm{m}^{-3}$ for $35,000 \mathrm{ppm}$ seawater at a recovery of $50 \%$ [2].

For further energy savings in the desalination stage of RO, improved process design may offer considerable benefits. Particularly interesting are batch processes and processes with increased stages, which theoretically can approach the thermodynamic minimum energy of separation [1113]. While these processes are promising, their energy requirements are largely unclear. Previous analyses neglected process inefficiencies [11-13], which are critical for an accurate comparison of the various process configurations. A rigorous quantitative analysis of the energetic requirements of the various RO configurations is clearly needed.

In this study, we quantitatively compare the energy of the desalination stage for batch and semi-batch RO processes with conventional RO processes and RO processes with increased staging. We first introduce the considered process configurations, highlighting particularly important components. We then discuss the minimum energies theoretically possible to illustrate the fundamental constraints stemming from each configuration. After establishing the intrinsic minimum energy requirements, we derive analytical approximations to gain insight into how realistic process inefficiencies, such as frictional pressure loss, affect the total energy efficiency for each configuration. Next, we rigorously compare the energetic requirements of the different 
processes using module-scale, numerical modeling. Lastly, we discuss other factors such as capital costs, operational experience, and process robustness that may distinguish the different process configurations.

\section{Considered process configurations}

\subsection{Staged reverse osmosis}

In current desalination facilities, both SWRO and BWRO are operated in a once-through fashion, meaning the concentrated brine is disposed of without recycle after passing through the membrane modules. SWRO is typically operated in a single stage (Fig. 1A) with recoveries of $35-50 \%$ [3]. ERDs are crucial in SWRO for decreasing energy usage by recovering energy from the high-pressure brine [14]. Essentially all new SWRO plants employ ERDs, typically isobaric work exchangers such as the DWEER device from Flowserve Corporation (Irving, TX) and the PX Pressure Exchanger from Energy Recovery Inc. (San Leandro, CA), each of which can recover energy from the brine at efficiencies up to $98 \%$ [3, 15-17]. While ERDs have markedly decreased the energy consumption of SWRO plants, one-stage RO is still far from optimal from an energy perspective. For the case discussed earlier with 35,000 ppm seawater feed and 50\% recovery, the thermodynamic minimum specific energy of desalination is $1.1 \mathrm{kWh} \mathrm{m}^{-3}, 0.5 \mathrm{kWh}$ $\mathrm{m}^{-3}(\sim 30 \%)$ less than the minimum for one-stage operation with a perfectly efficient ERD [2].

BWRO is typically operated with two or three stages (Fig. 1B) with recoveries of $75-90 \%$ that highly depend on the feed composition (e.g., the initial concentrations of TDS and sparingly soluble salts) [3]. Industrial wastewater treatment similarly uses up to three stages. The least energy-efficient staged configuration utilizes pumps ahead of only the first stage, with feed hydraulic pressures greater than that of the exiting brine. In this configuration, the decreasing number of pressure vessels in parallel in later stages serves mostly to modulate the cross-flow velocity, and energetically this configuration is similar to one-stage RO with no ERDs [18]. Increased efficiency is gained through the usage of inter-stage booster pumps, which allows for operation at multiple hydraulic pressures to not only save energy but also balance water fluxes (e.g., to mitigate fouling in lead membrane elements). The most energy-efficient staged configuration incorporates both inter-stage booster pumps and ERDs, including isobaric ERDs and turbochargers [19]. The decision to include inter-stage pumps and ERDs is made on the basis 
of life-cycle cost analyses, i.e., whether the savings in energy costs outweigh the increased capital investment. Inclusion of inter-stage pumps and ERDs is most favorable for high-recovery processes treating high-salinity feeds.

\subsection{Batch and semi-batch reverse osmosis}

In contrast to conventional, finite-staged $\mathrm{RO}$ processes, batch processes can in theory approach the thermodynamic minimum energy of desalination. Many configurations have been proposed [12, 13, 20-23]. The simplest batch RO process recycles the brine to an unpressurized feed tank until the target recovery or tank concentration is reached [24, 25]. Such a process is common in the process industries, e.g., for the concentration of specialty chemicals [24]. However, since the energy of the high-pressure brine is wasted, this process is not energy efficient and even requires a heat exchanger to chill the feed due to heat dissipation from the rapid depressurization of the recycled brine [24].

To realize the ideal energy consumption requirements of batch $\mathrm{RO}$, the tank/membrane recirculation loop should remain fully pressurized, with the pressure in the loop increasing as the osmotic pressure of the contents of the loop increases (Fig. 1C). Meeting this condition though is challenging due to the incompressibility of water and the decreasing volume of retained solution over the course of a cycle. Incorporating pistons (variable-volume feed tank) or a pressurized headspace might overcome this technical challenge, but would have substantial cost, complexity, and/or safety concerns. To date, a true batch RO system has yet to be demonstrated in which the system remains pressurized during operation. In other words, an energy-efficient, scalable batch $\mathrm{RO}$ process has yet to be realized.

One relatively straight-forward modification that may lead to performance approaching that of an ideal batch RO process would be to incorporate ERDs into the simple batch RO process using an unpressurized feed tank discussed above (Fig. 1D). Although ERDs, particularly turbochargers due to their chemical robustness, have been incorporated in batch RO processes in chemical processing plants [24], employment of ERDs in batch RO for water-treatment applications is unexplored. We envision batch RO with ERDs for near-continuous water treatment as having a few key steps. During concentration, feed is drawn from the feed tank, pressurized using a high-pressure pump and ERD, and pumped into the membrane modules. Energy in the brine is continually recovered using the ERD in a similar fashion as one-stage RO, except that the brine is recycled to the feed tank instead of being directed for disposal. During a 
given cycle, the feed pressure is increased to maintain constant flux until a target recovery or concentration in the feed tank is reached. At this point the membrane loop is depressurized and the feed is switched to a fresh feed tank to start a new cycle and minimize downtime, resulting in a near-continuous process. While the feed in the second tank is being concentrated, the original tank is emptied of brine and filled with fresh feed. A small number of large-volume tanks would be used to save on the costs of the tanks and to centralize operations, which can further decrease costs [26]. Isobaric ERDs such as the DWEER device would likely be suitable for this application, since the DWEER can operate at high efficiency even with variable pressure $[15,19]$. 188 At perfect ERD efficiency, batch RO with ERDs would obtain the same energetics as the pressurized (ideal) batch RO process.

Semi-batch RO is another practical alternative that maintains some features of a batch RO 191 process. In semi-batch $\mathrm{RO}$, pressure is maintained in the feed/membrane loop by adding fresh feed at the same flow rate as the permeate exiting the system (Fig. 1E) [27, 28]. This configuration has been commercialized by Desalitech, Inc. (Newton, MA) under the name closed-circuit desalination. While semi-batch RO is now a proven process, it is not a true batch process; the fresh feed continuously dilutes the recycled brine, leading to an increased minimum energy as discussed in Section 3.

197 Semi-batch RO has been applied to both SWRO and BWRO, and employs cycles of 198 concentration and depressurization similar to that proposed above for batch RO with ERDs. Although semi-batch RO technically does not need ERDs to recover energy, for SWRO applications an external pressure vessel is typically used as an isobaric work exchanger, similar in working principle to the DWEER and PX Pressure Exchanger devices [27, 29]. During the concentration cycle, the vessel is filled with fresh feed using a low-pressure pump. At the start of the next cycle, the high-pressure pump pushes the brine from the recirculation loop into the external vessel, pressurizing the fresh feed in the external vessel to the starting pressure of the cycle and pushing the feed into the membrane module. Using a pilot-scale test plant with this setup, Desalitech reported specific energy consumptions of $1.9-2.2 \mathrm{kWh} \mathrm{m}^{-3}$ for $41000 \mathrm{ppm}$ seawater feed at $47.5 \pm 1.5 \%$ recovery [27]. Similar energy consumptions $\left(1.9-2.2 \mathrm{kWh} \mathrm{m}^{-3}\right)$ were also reported for $\sim 35000$ ppm seawater feed at $42-53 \%$ recovery [29]. 
212 Figure 1. Simplified reverse-osmosis (RO) process designs considered in this study: (A) one-stage RO, (B) multi-stage RO, (C) relatively impractical batch $\mathrm{RO}$ process that utilizes a pressurized feed tank and can be considered ideal from an energetics perspective as the energy of the brine is fully retained, (D) relatively practical batch RO process utilizing energy recovery devices (ERDs) and an unpressurized feed tank, and (E) semi-batch RO process commonly known as closed-circuit desalination. ERDs are illustrated as the isobaric work exchanger DWEER device (Flowserve Corp., Irving, TX), but may take other forms as well. In (B), three-stage RO is depicted in the most energy-efficient form, i.e., with interstage booster pumps and an ERD. A similar diagram would apply for less efficient variations, in which the ERD and/or inter-stage booster pumps would not be employed. In (C), a variable-volume tank is depicted to maintain pressure within the tank. A pressurized headspace would be another alternative. In the transient processes $(\mathrm{C}-\mathrm{E})$, only the concentration cycle is shown. Valves are not shown in any of the diagrams. HPP: high-pressure pump; BP: booster pump; LPP: low-pressure pump.

\section{Minimum energy of conventional and alternative process designs}

Before considering the effects of process inefficiencies on the energetics of the different process configurations shown in Fig. 1, we first consider the minimum specific energy that is theoretically possible for each process, following previously published works [11-13]. The difference in minimum energy between processes gives insight into the intrinsic effects that configurations have on energy consumption. Our previous analysis explored minimum energies in greater detail, but did not include batch RO [11]. Following the format of our previous analysis, we show the energies as a function of the system recovery, $R$, and express the specific energies relative to the initial feed osmotic pressure, $\pi O$, which is the thermodynamic minimum that salt is $100 \%$ rejected, ERDs are used in all relevant processes, there are no process inefficiencies, and the osmotic pressure can be expressed using the van't Hoff equation as a linear function of salt concentration.

238 Conventional once-through processes have either one stage or multiple stages in series. In one-stage $\mathrm{RO}$, the minimum hydraulic pressure throughout the module is the osmotic pressure of 
240 the exiting brine; thus, the minimum specific energy, $S E$ 1, $\min$, is simply the osmotic pressure of

241 the exiting brine $[11,12]$ :

$\operatorname{SE} 1, \min (R)=\pi 01-R$

242 Staging allows for operation at multiple hydraulic pressures, meaning that early stages run at a 243 lower pressure than is required for one-stage operation. While staging can be arranged in an 244 infinite set of ways, we specify here that each stage have the same per-stage recovery. For RO 245 with $N$ stages, the minimum specific energy, $S E N$, $\min$, is the following [12]:

$\operatorname{SEN}, \min (R)=\pi O R N 1-R 1 / N-N$

246 Unlike staged, once-through processes, the osmotic pressure during a batch RO cycle is not 247 constrained by the system recovery. To calculate the minimum specific energy of batch RO, we 248 note that the minimum hydraulic pressure at a given point in a concentration cycle is the osmotic 249 pressure at the given concentration. Therefore, the minimum specific energy in batch RO, $250 S E B, \min$, is by definition the thermodynamic minimum energy of separation, $S E m i n$, which is 251 the volume-weighted integral of the osmotic pressure $[11,12]$ :

$S E B, \min (R)=S \operatorname{Emin}(R)=\pi O R \ln 11-R$

252 Batch RO is not the only process that can theoretically match the thermodynamic minimum 253 energy; the minimum specific energy for a staged process with an infinite number of stages $254(N \rightarrow \infty)$ is similarly equal to $S E \min$.

255 Semi-batch RO (or closed-circuit RO) is not a true batch process. The minimum specific 256 energy for semi-batch RO, SESB, min, exceeds that of batch RO, and can be given as $[11,12]$ :

$S E S B, \min =\pi 02-R 21-R$

257 The increased energy consumption in semi-batch RO is discussed below.

FIGURE 2

Figure 2. (A) Normalized minimum specific energies for the reverse-osmosis (RO) process designs 262 considered in this study. Specific energies, $S E$, are normalized to the initial osmotic pressure of the feed, $\pi O$. Gray shaded area corresponds to the minimum thermodynamic energy of separation. (B) Normalized 
permeate volumes for semi-batch and batch RO processes. Permeate volumes for semi-batch and batch $\mathrm{RO}$ are normalized to the volume in the circuit and the volume in the feed tank, respectively.

The minimum specific energies for one-stage, two-stage, three-stage, semi-batch, and batch RO are shown versus recovery in Fig. 2A. For all processes, the minimum energy occurs at the limit of zero recovery, which of course is only true in the ideal case of no process inefficiencies. One-stage RO requires the greatest minimum energy, and adding additional stages markedly decreases the minimum energy requirements. The most interesting result is the disparity between semi-batch RO and batch RO: semi-batch RO requires much greater minimum energy than batch RO at high recoveries. Thermodynamically, this increased energy is due to entropic losses; the mixing of high-concentration recycled brine with low-concentration feed in semi-batch RO generates high levels of entropy [11]. In contrast, the recycled brine in batch RO has a similar concentration to the contents of the feed tank, resulting in relatively low entropy generation in practice and zero entropy generation in the limit of zero recovery per pass through the membrane modules.

An alternative mathematical explanation for the differing energies can be given by considering how recovery is defined in the two transient processes. In batch RO, recovery is given as $R=V P / V T$, where $V P$ is the cumulative permeate volume and $V T$ is the volume of the

feed tank. The feed tank volume can be sized to greatly exceed the volume in the membrane modules, and essentially all of the contents of the feed tank can theoretically be recovered. In contrast, recovery in semi-batch RO is related to both the initial feed volume in the circuit, $V C$, which cannot be recovered, and the cumulative feed input during a cycle, which is equal to the cumulative permeate volume. Recovery is thus given as $R=V P /(V P+V C)$. When $V P V C$ (i.e.

287 low recovery), $V P$ can be neglected in the denominator, resulting in batch-like energetics. At high recoveries, however, $V P$ becomes important, and a much greater relative permeate volume is needed to increase recovery in semi-batch $\mathrm{RO}$ when compared with batch RO (Fig. 2B). The effect of the increased relative permeate volume on energy consumption is further magnified by 291 the relatively high osmotic pressures (and corresponding hydraulic pressures) when operating in 292 the high-recovery regime. Combined together, the two effects yield a sharp increase in minimum 293 specific energy for semi-batch RO above a recovery of $\sim 0.7$ (Fig. 2A). 


\section{Analytical approximations of specific energy requirements}

While the minimum energy discussion in Section 3 provides insight into the intrinsic effects that process configurations have on process energetics, the inclusion of process inefficiencies is critical to enable realistic comparisons of the different configurations. These inefficiencies, such as frictional losses, pump efficiency, and ERD efficiency, may not affect each configuration equally. In this section, we derive simple analytical approximations to determine how process inefficiencies affect the energetics of each process design. For staged RO processes, the highest efficiency configuration was considered wherein inter-stage booster pumps and ERDs are incorporated.

\subsection{Process inefficiencies}

Frictional pressure losses can be separated into two types: losses in the membrane elements and losses in the related piping and valves. Pressure loss in the membrane elements is due to friction in the feed channels, permeate channels, and anti-telescoping devices that separate each element. Neglecting the relatively minor loss within the permeate channels, we combine the pressure losses in the spacer-filled feed channels and between elements into a lumped channel pressure loss, $\triangle$ Pchan. In our analysis, this pressure loss for one pass through a membrane pressure vessel was taken to be proportional to the recovery:

$\Delta$ Pchan $=r \Delta$ Pchanr $=1$

312 where $\Delta$ Pchanr $=1$ is the channel pressure loss if the per-pass recovery through the membrane 313 module, $r$, is extrapolated to 1 . The linear relationship between pressure loss and per-pass 314 recovery in Eq. 5 can be used because (for constant average permeate water flux, channel height, 315 and initial feed velocity) the recovery is proportional to the module length, which in turn is 316 proportional to the pressure loss in the feed channel. In addition, because increased length 317 signifies more membrane elements, pressure loss in anti-telescoping devices between each 318 element should also scale with length and recovery. It should be noted though that Eq. 5 neglects 319 the decrease in feed velocity along the module, which in real systems would result in a decreased 320 rate of pressure loss as feed travels down the module. This effect is accounted for in the 
321

322

323

324

325

326

327

328

329

330

331

332

333

334

335

336

337

338

339

340

341

342

343

344

345

346

347

348

349

numerical modeling in Section 5. The frictional loss in the feed channels at full recovery can be normalized to the feed osmotic pressure, with $\Delta$ pchanr $=1=\Delta$ Pchanr $=1 \pi 0$.

The second type considered is the circulation pressure loss, $\Delta$ Pcirc, which broadly includes all frictional losses within related piping and valves during RO operation. This pressure loss can also be normalized to the feed osmotic pressure, with $\Delta p c i r c=\Delta P \operatorname{circ} \pi O$. Unlike the channel pressure loss, circulation pressure loss does not scale with recovery. We instead relate the circulation pressure loss to the cumulative flow that enters and exits each bank of membrane pressure vessels (e.g., each stage in staged RO), since for each pass through a pressure vessel, a volume of liquid must also pass through all of the associated pipes, valves, and (if applicable) booster pumps. Although the pipe configuration will differ slightly between process configurations, the magnitude of the pressure loss with each pass through the pipe network should be roughly similar. As such, circulation pressure losses are expected to be larger for multi-staged RO compared to one-stage RO due to the needed passes through the inter-stage piping. Similarly, circulation pressure losses are expected to be larger in batch and semi-batch operations because of the large flow of recycled brine. Even though circulation pressure losses in batch and semi-batch RO are small on a per-pass scale, the cumulative effects can be substantial, as discussed later.

Further process inefficiencies include the efficiencies of the high pressure pump, $\eta p$, and the energy recovery devices, $\eta E R D$. Although each configuration uses multiple pumps (Fig. 1) that can differ in efficiency [27], the same pump efficiency is assumed for all pumps. In addition, hydraulic over-pressures are not included for simplicity, meaning that the hydraulic pressures required for inducing flux were assumed to be equal to the osmotic pressure of the brine (retentate) leaving the membrane module. Effects of over-pressure are considered in the numerical modeling in Section 5.

\subsection{Analytical specific energy approximations}

The analytical approximations for the specific energies, $S E$, of each configuration are listed below, and bear some resemblance to the corresponding minimum energy equations in Section 3. Only one equation is included for batch RO. The specific energy of ideal batch RO can be obtained by simply setting $\eta E R D$ to 1 . All derivations are included in Appendix A. 
One-stage: $\quad S E 1 \pi O=1 \eta P 1 R 11-R-\eta E R D+\Delta p \operatorname{circ} R+\Delta p \operatorname{chanr}=1$

$N$ stages: $\quad S E N \pi O=1 \eta P 1 R N 1-R 1 N+1-N-\eta E R D+\Delta$ pcircr $+\Delta$ pchanr $=1$

Batch: $\quad S E B \pi 0=1 \eta P 1 r R 11-r-\eta E R D \ln 11-R+\Delta p c i r c r+\Delta p c h a n r=1$

Semi-batch: $\quad S E S B \pi 0=1 \eta P 2-R 21-r 1-R+\Delta p c i r c r+\Delta p c h a n r=1$

350

351

352

353

354

355

356

357

358

359

360

361

362

363

364

365

366

367

368

369

370

371

372

373

374

Eqs. 6-9 include the per-pass recovery, $r$, which is the recovery obtained for each stage in multistage RO and for each pass through the module in batch and semi-batch RO. In one-stage RO, the per-pass recovery equals the system recovery, $R$. As defined in Section 3, the per-pass recovery in multi-stage RO is set so that the recovery is the same in each stage, and is related to the total system recovery with $r=1-1-R 1 N$. The per-pass recovery in batch and semi-batch RO will be discussed further later.

Several relationships between process inefficiencies and specific energy requirements can be immediately gleaned from the derived approximations. First, specific energies are all reciprocally related to the pump efficiency. Increased pump efficiency would thus benefit all processes equally. In contrast, the efficiency of the energy recovery devices affects the processes differently. The ERD efficiency is simply subtracted from the energies in one-stage and multistage RO, whereas it is involved in the energetics of batch RO in a more complex fashion.

The frictional losses within the membrane channels also affect each process equally, which is a surprising result considering that in batch and semi-batch processes the feed-stream passes through the membrane module several times before the completion of a given cycle. This finding can be explained by the linear relationship in Eq. 5 between channel pressure loss and recovery. Since the per-pass recoveries in batch and semi-batch RO are much lower than the system recovery, the per-pass channel pressure loss is similarly small.

In contrast to the channel pressure loss, the circulation pressure loss differs between configurations and is inversely related to the per-pass recovery. While this relationship is a somewhat simple result, it is physically logical; the total circulation pressure loss occurs for a given volume passing through the pipe network one time, which when divided by the per-pass recovery of that given volume yields the contribution to the specific-energy requirements. For a small per-pass recovery (as can occur in batch and semi-batch processes), the effects of the circulation pressure loss are therefore magnified substantially, as shown later. 
By differentiating the specific energy approximations for batch and semi-batch RO (Eqs. 8-9) with respect to $r$, the optimal per-pass recoveries can be derived. For batch RO, the optimal recovery, $r B *$, is the following:

$r B *=1+\Delta p \operatorname{circ} 1 R \ln 11-R-\eta E R D-1+\Delta p \operatorname{circ} 1 R \ln 11-R-\eta E R D \eta E R D-\Delta p \operatorname{circ} 1 R \ln$ $11-R$

Interestingly, the ratio of the absolute circulation pressure loss, $\Delta$ Pcirc, to the thermodynamic minimum energy of separation, SEmin (Eq. 3), at the given system recovery partially determines the optimal per-pass recovery. The ERD efficiency also plays a role.

For semi-batch RO, the optimal recovery, $r S B \star$, is the following:

$r S B *=11+2-R 21-R \Delta p c i r c$

383 Here, only the circulation pressure loss and system recovery determine the optimal per-pass recovery. As expected, the optimal per-pass recoveries for batch and semi-batch RO are zero at the limit of a perfectly efficient process (i.e., $\triangle p c i r c \rightarrow 0, \eta E R D \rightarrow 1$ ).

\subsection{Process trends and critical parameters}

Using the analytical approximations in Eqs. 6-11, the normalized specific energies are shown for each configuration in Fig. 3A. Some trends remain similar to those of the minimum energy curves. For example, ideal batch RO uses by far the least energy at high recoveries, and consumes less energy than semi-batch RO for all recoveries. Increased staging also maintains substantial energy reductions when compared to one-stage RO, and yields similar energy

\section{FIGURE 3}

Figure 3. Analytical approximations for (A) the specific energies, $S E$, normalized to the feed osmotic 
normalized circulation pressure loss, $\Delta$ pcirc, of 0.01 , normalized pressure loss in the membrane elements

399 for full per-pass recovery, $\Delta p c h a n r=1$, of 0.1 , and pump efficiency, $\eta P$, of 0.8 . For one- and two-stage

$400 \mathrm{RO}$, the energy recovery device efficiency, $\eta E R D$, was set to 0.98 . In (A), the gray shaded area indicates

401 the thermodynamic minimum specific energy required for desalination. Ideal batch RO curves were 402 calculated by setting $\eta E R D$ to 1 .

403 There are several noticeable changes that transpire when process inefficiencies are included. 404 All of the curves are shifted up considerably from the minimum energy curves in Fig. 2A, mostly 405 due to the pump efficiency, which affects each configuration equally and was set here to 0.8 . In 406 addition, the minimum for staged configurations is no longer at the limit of zero recovery, 407 instead occurring at $15-30 \%$ recovery when the ERD efficiency is set to 0.98 . The minimum 408 shifts to even higher recoveries for lower ERD efficiencies, as has been shown previously [30]. 409 In contrast, the minimum for batch and semi-batch processes remains at the limit of zero 410 recovery, mostly because the per-pass recovery can be operated independently of the system 411 recovery. Lastly, for the normalized circulation pressure setting of 0.01 shown in Fig. 3A, ideal 412 batch RO is comparable in efficiency to three-stage RO for recoveries of $30-50 \%$. In the 413 minimum energy curves in Fig. 2A, ideal batch RO is more efficient than three-stage RO at all 414 recoveries.

415 The analytical approximations also show that the ERD efficiency is very critical for batch 416 processes. Decreasing the efficiency from 1 (ideal batch) to 0.98 and 0.95 sharply increases the 417 required energy, and also increases the optimal per-pass recovery as shown in Fig. 3B. The 418 effects of ERD efficiency are further illustrated in Fig. 4A, which compares staged 419 configurations and batch RO at a recovery of 50\%. As ERD efficiency increases, the energy 420 required for batch $\mathrm{RO}$ falls at a much faster rate than for staged systems, for which the required 421 energy decreases at a consistent, linear rate. In order to consume less energy in batch than one422 stage RO, ERD efficiencies greater than 0.95 are required. In order to consume less energy than 423 two-stage RO, ERD efficiencies greater than 0.995 are required for batch RO. Clearly, in order 424 for batch RO with ERDs to be energy-efficient, ERDs must be able to achieve efficiencies 425 towards the high end of what is achieved in one-stage SWRO. Since there is no data for such a 426 process, it is unclear whether such efficiencies would be possible. 
Fig. 4B shows how circulation pressure losses affect the specific energy of each process configuration. It is worth emphasizing that the shown pressure loss is normalized to the feed osmotic pressure. Thus, increased normalized circulation pressure loss could stem from piping design (e.g., type and length of pipes, pipe connectors and valves) or from a relatively low feed salinity. Circulation pressure loss is therefore expected to impact BWRO more significantly than SWRO, since the absolute pressure losses should be similar between the two processes. In terms of trends in Fig. 4B, the circulation pressure losses clearly impact batch and semi-batch processes most significantly, which is due to the low per-pass recoveries $(r<0.10)$ in these processes (Fig. 3B). The contribution to specific energy consumption rises steeply at low circulation pressure loss, and levels off slightly at higher circulation pressure losses due to the increased optimal per-pass recoveries. Increased staging also leads to a greater effect of circulation pressure loss, as indicated by the slopes of the two-stage and three-stage curves. The curves for staged processes are linear because the per-stage recoveries are fixed.

\section{FIGURE 4}

Figure 4. Effect of (A) energy-recovery-device efficiency, $\eta E R D$, and (B) normalized circulation

444 pressure loss in the system piping, $\Delta$ pcirc, on the energy requirements of conventional and alternative reverse-osmosis (RO) processes. Curves were calculated using Eqs. 6-11 with a total recovery, $R$, of 0.5, normalized feed-channel pressure loss at full recovery, $\Delta$ pchanr $=1$, of 0.1 , and pump efficiency, $\eta P$, of 0.8. In (A), $\Delta$ pcirc was set to 0.01 . In (B), batch RO is ideal, with an energy recovery device efficiency, $\eta E R D$, of 1. For staged RO, $\eta E R D$ was set to 0.98. $S E$ : specific energy; $\pi O$ : feed osmotic pressure.

The analytical work in this section was largely intended to provide insight into trends, or in other words, how process parameters affect process energetics. Important insights include the criticality of ERD efficiency in batch RO, the relative importance of circulation pressure loss in piping for staged, batch, and semi-batch RO, and the equal impacts that channel pressure loss and pump efficiency have on the different configurations. The analytical approximations also provided semi-quantitative energetic comparisons of the different configurations, as shown in Fig. 4A, with indications that two-stage and semi-batch RO might save energy at recoveries 
around 50\%, while three-stage RO and batch RO with ERDs might save energy at high recoveries of $80-90 \%$. However, as mentioned earlier, hydraulic over-pressure and variable feed velocities were neglected in these approximations. Numerical modeling with controlled process parameters (e.g., water flux) is needed for more accurate comparison. Such modeling is conducted and discussed in the next section.

\section{Module-scale analysis of alternative process designs}

Numerical modeling enables us to build on the analytical work presented Section 4 in two main ways. Firstly, we are able to calculate the hydraulic pressure required to achieve a specified average water flux across a membrane module, accounting for the effects of concentration polarization, membrane resistance, hydrodynamics, and varying solution conditions. This to be the hydraulic pressure in our analytical approximations in Section 4. Secondly, numerical modeling enables us to determine how the feed flow rate, velocity, and solute concentration vary along the membrane module. These module-scale profiles can then be used with well-established

473 The numerical calculations presented in this section are based on a one-dimensional finite element model of the membrane module. After incorporating the efficiencies of the pumps and energy recovery devices, as well as a circulation pressure loss, the total energy consumptions are calculated for each process from the numerically calculated hydraulic pressure and frictional 477 pressure loss.

\subsection{Calculating the hydraulic pressure required to achieve a target average water flux}

479 Eqs. 12 and 13 describe how the feed flowrate, $Q F$, and solute concentration, $c F$, vary with membrane area, $A m^{\prime}$, along the membrane module:

$$
\begin{gathered}
d Q F d A m^{\prime}=-J w \\
d Q F c F d A m^{\prime}=-J S
\end{gathered}
$$

481 To calculate the hydraulic pressure, $\Delta P$, required to achieve a certain water recovery, $R$, with a 482 specified average water flux, $J w$, we solve Eqs. 12 and 13 simultaneously, subject to two 483 boundary conditions. First, at the start of the module $\left(A m^{\prime}=0\right): Q F=Q F O$ and $c F=c F O$, while at 
484 the end of the module $\left(A m^{\prime}=A m\right): Q F=Q F 01-R$. The specified average water flux constrains 485 the total membrane area: $A m=R Q F O / J w$. Eqs. 12 and 13 are solved using an iterative multistep 486 numerical method until both boundary conditions are met.

487 To integrate Eqs. 12 and 13 we calculate the water flux, $J w$, and solute flux, $J_{S}$, at each step

488 along the membrane module. The water and solute fluxes are given by [31, 32]:

$J w=A \Delta P-\pi F, m-\pi P$

$J_{S}=B c F, m-c P$

where $A$ and $B$ are the water and solute permeability coefficients of the membrane, respectively,

$490 \pi F, m$ is the osmotic pressure of the feed at the membrane surface, $\pi P$ is the osmotic pressure of

491 the permeate, $c F, m$ is the solute concentration at the membrane surface, and $c P$ is the solute

492 concentration of the permeate. An expression for $c F, m$ can be found by solving the film model to

493 account for concentration polarization [31]:

$c F, m=c F, b \exp J w k F-J S J w \exp J w k F-1$

494 where $c F, b$ is the bulk solute concentration on the feed-side of the membrane and $k F$ is the feed-

495 side mass transfer coefficient. The mass transfer coefficient varies along the module depending 496 on the local feed flow rate, and is calculated using a Sherwood-Reynolds-Schmidt correlation 497 [33]. More details are provided in Appendix B. The concentration in the permeate, $c P$, is given 498 by:

$c P=J_{S} J w$

499 The dependence of the osmotic pressure on solute concentration is calculated using data from

500 OLI Systems (Cedar Knolls, NJ).

5015.2 Calculating the frictional pressure loss in the feed-side membrane channel

502 The frictional pressure loss in the feed-side membrane channel is calculated using an 503 established semi-empirical correlation [34, 35]:

$d P \operatorname{chand} L=0.8 \operatorname{Re}-0.19 \operatorname{Re} 2 \rho v 2 \mathrm{dh} 3 L$ 
504 where dPchan is the infinitesimal frictional pressure loss in the channel, $R e$ is the Reynolds

505 number, $\rho$ is the fluid density, $v$ is the kinematic viscosity, $L$ is the length of the membrane

506 channel, and $d h$ is the hydraulic diameter of the spacer-filled channel. The correlation used has

507 been derived from computational fluid dynamics studies of flow through a spacer-filled 508 membrane channel [34]. It has been shown to agree with other modeling and experimental 509 studies for typical flow conditions [35].

510 The Reynolds number, density, and kinematic viscosity of the feed flow change along the 511 membrane module as water permeates through the membrane and solutes are retained [36]. To 512 account for these variations $d P c h a n / d L$ is calculated for each step in the multistep numerical 513 integration of Eqs. 12 and 13 described in Section 5.1, and summed to yield a total channel 514 pressure loss, $\triangle$ Pchan. An initial feed channel velocity of $0.2 \mathrm{~m} \mathrm{~s}^{-1}$ is assumed.

\subsection{Calculating the specific energy of staged, batch, and semi-batch RO}

516 Expressions for the specific energies, $S E$, of one-stage, two-stage, three-stage, batch, and 517 semi-batch RO are listed below in Eqs. 19-23, respectively. Eqs. 19-23 are broadly similar to 518 Eqs. 6-9 in Section 4.2, which were derived analytically as shown in Appendix A by setting the 519 hydraulic pressure across a membrane module equal to the osmotic pressure of the exiting 520 retentate. In Eqs. 19-23, the hydraulic pressure terms, $\Delta P$, and the frictional pressure loss in the 521 channel, $\triangle$ Pchan, are calculated numerically using the model described in Sections 5.1 and 5.2.

Onestage:

$$
S E 1=1 \eta P 1 R 1-1-R \eta E R D \Delta P 1+\Delta P \operatorname{circ} R+\triangle P c h a n
$$

Twostage:

$$
S E 2=1 \eta P 1 R \Delta P 1+1-r \Delta P 2-\Delta P 1-1-R \eta E R D \Delta P 2+\Delta P c i r c r+\Delta P c h a n
$$

Three-

$$
S E 3=1 \eta P 1 R \Delta P 1+1-r \Delta P 2-\Delta P 1+1-r 2 \Delta P 3-\Delta P 2-1-R \eta E R D \Delta P 3+\Delta P c i r c r+\Delta P c h a n
$$

stage:

Batch: $\quad S E B=1 \eta P 1 r t B 1-1-r \eta E R D O t B \Delta P d t+\triangle P c i r c r+1 t B O t B \Delta$ Pchan $d t$

Semi-

batch:

$$
S E S B=1 \eta P 1 t B O t B \Delta P d t+\Delta P c i r c r+1 t B O t B \Delta P c h a n d t
$$


where $\Delta P i$ is the hydraulic pressure applied across stage $i$. The water recovery in each stage of an $N$-stage RO process is again $r=1-1-R 1 / N$. To calculate the specific energies of batch (Eq. 21) and semi-batch (Eq. 22) RO, we need to compute the integral of hydraulic pressure with respect to time, $t$, over a batch cycle, i.e., from $t=0$ to $t=t B$, where $t B$ is the total cycle time. These integrals are calculated using a numerical method with multiple steps in $t$.

\subsection{Quantitative comparison of energy required for each process configuration}

The parameters used for our module-scale analysis are presented in Table 1, with further details provided in Appendix B. The membrane water permeability coefficient is based on that of state-of-the-art thin-film composite polyamide membranes [9]. Typical feed channel and spacer geometries were selected from the literature [34]. The solute was modeled as sodium chloride for both seawater and brackish water. The membrane solute permeability coefficient was set to zero (i.e., salt rejection of $100 \%$ ) to ensure identical constraints were placed on the different configurations. Because permeating solute decreases the hydraulic pressure (and corresponding energy) needed to achieve the target water flux (Eq. 14), variations in permeate solute concentration can affect the calculated energy requirements.

Table 1. Parameters used in the module-scale analysis of seawater and brackish water reverse 539 osmosis.

\begin{tabular}{|c|c|c|}
\hline \multicolumn{2}{|l|}{ Property } & Value \\
\hline \multicolumn{2}{|c|}{ Membrane water permeability coefficient, $A$} & $3.0 \mathrm{~L} \mathrm{~m}^{-2} \mathrm{~h}^{-1} \mathrm{bar}^{-1}$ \\
\hline \multicolumn{2}{|c|}{ Membrane solute permeability coefficient, $B$} & $0.0 \mathrm{~L} \mathrm{~m}^{-2} \mathrm{~h}^{-1}$ \\
\hline \multicolumn{2}{|l|}{ Average water flux, $J w$} & $15 \mathrm{~L} \mathrm{~m}^{-2} \mathrm{~h}^{-1}$ \\
\hline \multicolumn{2}{|l|}{ Pump efficiency, $\eta P$} & 0.80 \\
\hline \multirow{2}{*}{$\begin{array}{l}\text { Energy recovery device } \\
\text { (ERD) efficiency, } \eta E R D\end{array}$} & Batch RO, Staged RO & 0.98 \\
\hline & Ideal (pressurized tank) batch RO & 1.00 \\
\hline \multicolumn{2}{|l|}{ Initial feed channel velocity } & $0.2 \mathrm{~m} \mathrm{~s}^{-1}$ \\
\hline \multicolumn{2}{|l|}{ Feed channel height } & $0.77 \mathrm{~mm}$ \\
\hline \multicolumn{2}{|l|}{ Spacer porosity } & 0.89 \\
\hline
\end{tabular}




\begin{tabular}{|l|l|r|}
\hline \multicolumn{2}{|l|}{ Feed channel hydraulic diameter, $d h$} & $0.95 \mathrm{~mm}$ \\
\hline $\begin{array}{l}\text { Initial solute concentration } \\
\text { (modeled as sodium } \\
\text { chloride), } c F 0\end{array}$ & Seawater & $0.6 \mathrm{~mol} \mathrm{~L}^{-1}$ \\
\cline { 2 - 3 } & Brackish water & $0.1 \mathrm{~mol} \mathrm{~L}^{-1}$ \\
\hline $\begin{array}{l}\text { Circulation frictional } \\
\text { pressure drop, } \triangle \text { Pcirc }\end{array}$ & Moderate frictional loss case & 0.1 bar \\
\cline { 2 - 3 } & High frictional loss case & 0.5 bar \\
\hline
\end{tabular}

The per-pass water recovery, $r$, plays an important role in determining the specific energy of

542 batch and semi-batch RO. In Section 4.3, we derived analytical expressions for the optimal perpass recovery, $r$ * for batch RO (Eq. 10) and semi-batch RO (Eq. 11). These analytically derived

$544 r$ * values slightly underestimate the numerically determined $r$ * values, as shown in Fig. B.1 in

545 Appendix B. However, our numerical analysis showed that the specific energy of batch and semi-batch RO is not heavily dependent on $r$, provided that $r \approx r *$. Consequently, we have

547 calculated $r$ using our analytical expressions for $r *$ in the remainder of this study.

548 Fig. 5A shows how the specific energy of SWRO varies with water recovery for each process configuration. The trends observed in Fig. 5A are similar to those seen in Fig. 3A. Ideal batch RO has the lowest specific energy across the entire range of recoveries. However, as the ERD efficiency, $\eta E R D$, drops from 100\% (i.e., ideal, pressurized batch RO) to $98 \%$, the energy requirement of batch RO increases by approximately 15-20\%, making it less energy efficient than one-stage RO, two-stage, and semi-batch RO for recoveries lower than $45 \%, 68 \%$, and $63 \%$,

554 respectively. The decrease in ERD efficiency causes a substantial increase in the energy lost per555 pass and therefore increases the optimal per-pass recovery, $r B *$. For example, for a circulation 556 pressure loss, $\triangle$ Pcirc, of 0.1 bar, a drop in $\eta E R D$ from $100 \%$ to $98 \%$ leads to an increase in $r B$ * 557 from $4.7 \%$ to $13.0 \%$.

558 For a typical SWRO recovery of 50\%, Fig. 5B shows that all of the alternative processes can 559 save energy with respect to one-stage RO. With a moderate circulation pressure loss of 0.1 bar, 560 two-stage RO and semi-batch RO would reduce energy consumption by $15.3 \%\left(0.34 \mathrm{kWh} \mathrm{m}^{-3}\right)$ 
and $13.0 \%\left(0.29 \mathrm{kWh} \mathrm{m}^{-3}\right)$, respectively, compared to one-stage RO. Given the same parameters, 562 batch RO with ERDs is slightly less efficient, reducing energy consumption by $5.9 \%(0.13 \mathrm{kWh}$

$563 \mathrm{~m}^{-3}$ ), while ideal, pressurized batch RO would be the most efficient with a reduction of $19 \%$ $564\left(0.43 \mathrm{kWh} \mathrm{m}^{-3}\right)$.

565 As found in Section 4, higher circulation pressure losses affect batch and semi-batch 566 processes more than once-through processes. A comparison of batch and semi-batch RO with 567 two-stage RO in Fig. 5B highlights the impact of circulation pressure loss. For a $\Delta$ Pcirc of 0.1 568 bar, ideal batch RO requires $4.8 \%$ less energy than two-stage RO, whereas for a $\Delta$ Pcirc of 0.5 569 bar, ideal batch RO requires slightly more energy (1.4\%). Similarly, semi-batch RO uses 3.8\% 570 less energy than two-stage RO when neglecting $\triangle$ Pcirc, while it needs $2.7 \%$ and $9.0 \%$ more 571 energy for a $\Delta$ Pcirc of 0.1 bar and 0.5 bar, respectively.

\section{FIGURE 5}

Figure 5. Specific energy requirements calculated numerically through module-scale modeling for conventional and alternative seawater reverse osmosis (SWRO) processes. (A) Specific energy versus typical system recoveries, $R$, for a circulation pressure loss, $\Delta$ Pcirc, of 0.1 bar. The gray shaded area 578 indicates the thermodynamic minimum specific energy required for desalination. (B) Excess specific 579 energy (i.e., specific energy, $S E$, minus the thermodynamic minimum specific energy, $S E m i$ ) required 580 at a system recovery, $R$, of 0.5 for different circulation pressure losses. At this recovery, the 581 thermodynamic minimum specific energy is $1.15 \mathrm{kWh} \mathrm{m}^{-3}$. Parameters used in modeling are listed in 582 Table 1. Feed was modeled as $0.6 \mathrm{M}$ or $35000 \mathrm{ppm}$ sodium chloride. $\eta E R D$ : efficiency of energy 583 recovery device.

$584 \quad$ For BWRO, we consider a case with relatively high feed salinity $(0.1 \mathrm{M}$ or 5840 ppm sodium 585 chloride). For high-salinity feeds, energy requirements can be substantial, making energy 586 considerations relatively important. All trends observed are similar to those for a lower salinity 587 feed of 1000 ppm sodium chloride (data not shown), except that the absolute differences in 588 energy requirements between configurations are smaller in the low-salinity case. 
The trends observed for BWRO in Fig. 6A closely resemble those seen at the higher water recovery $(>75 \%)$ end of Fig. 3A. At these high recoveries that are typical for BWRO, energy losses due to the over-pressure at the beginning of a membrane module are more significant than the energy losses associated with the ERD and circulation pressure losses. Consequently, batch RO, even with a $\eta E R D$ of $98 \%$, and three-stage RO are the most promising BWRO processes from an energetic perspective. With a $\eta E R D$ of $98 \%$, batch RO has a lower specific energy than two-stage and three-stage RO for water recoveries in excess of $81 \%$ and $90 \%$, respectively. The sensitivity of the specific energy of batch RO to inefficiencies in the ERD is lower for BWRO than SWRO-reducing $\eta E R D$ from $100 \%$ (ideal batch RO) to $98 \%$ over relevant recoveries results in a $11-12 \%\left(0.06-0.08 \mathrm{kWh} \mathrm{m}^{-3}\right)$ increase in the specific energy in BWRO, compared with a $16-17 \%$ increase $\left(0.24-0.34 \mathrm{kWh} \mathrm{m}^{-3}\right)$ increase in SWRO. In contrast to batch RO, semibatch RO performs quite inefficiently throughout the relevant recovery range for BWRO. Of the curves shown, only one-stage RO requires more energy at high recoveries than semi-batch RO.

Fig. 6B highlights the energy requirements at a relatively high BWRO recovery of $90 \%$, for which energy consumption becomes relatively important. At this recovery and with a $\Delta$ Pcirc of 0.1 bar, batch and three-stage RO consume the least amount of energy: compared to two-stage RO, ideal batch RO saves $24.6 \%\left(0.22 \mathrm{kWh} \mathrm{m}^{-3}\right)$, while batch RO with ERDs and three-stage RO save $15.3 \%\left(0.14 \mathrm{kWh} \mathrm{m}^{-3}\right)$ and $14.0 \%\left(0.12 \mathrm{kWh} \mathrm{m}^{-3}\right)$, respectively. Semi-batch RO is significantly less efficient, consuming $37.5 \%$ more energy $\left(0.33 \mathrm{kWh} \mathrm{m}^{-3}\right)$ than two-stage RO. As described in Section 3, the increase in the energy consumption of semi-batch RO at high recoveries is due to entropy generated by the mixing of the highly concentrated recycled brine with the low concentration feed stream.

611 The above modeling assumed that staged RO operates using inter-stage booster pumps and 612 high-efficiency ERDs, which is the most highly efficient arrangement. As discussed in Section 2, 613 ERDs and even inter-stage booster pumps can be omitted in BWRO and industrial RO if life614 cycle cost analyses indicate that the energy savings do not outweigh the increased capital costs. 615 Exclusion of these components can substantially increase energy consumption. For example, for 616 the conditions shown in Fig. 6B (5840 ppm sodium chloride feed, 90\% recovery) at a moderate 617 circulation pressure loss of 0.1 bar, three-stage RO without inter-stage pumps and without ERDs 
618 (i.e., using only one bank of high-pressure pumps ahead of the first stage) requires $1.98 \mathrm{kWh} \mathrm{m}^{-3}$. 619 In this configuration, staging mainly affects the cross-flow velocity, and minimally affects the energy requirements since the feed pressure must exceed the brine osmotic pressure, as in one-

621 stage RO. In fact, the energy requirements are nearly identical to that of one-stage RO without 622 ERDs $\left(2.00 \mathrm{kWh} \mathrm{m}^{-3}\right)$. Incorporation of inter-stage pumps allows for operation at lower 623 hydraulic pressures in the earlier stages, decreasing the energy consumption more than $50 \%$ to $6240.95 \mathrm{kWh} \mathrm{m}^{-3}$. Inclusion of (isobaric) ERDs, in addition to inter-stage pumps, further decreases 625 the energy consumption to $0.77 \mathrm{kWh} \mathrm{m}^{-3}$, corresponding to the value shown in Fig. 6.

626 All energy comparisons discussed thus far between staged RO, batch RO, and semi-batch RO 627 also assumed that staged RO is operated most efficiently, i.e., with inter-stage pumps and 628 isobaric ERDs. Conclusions change if batch and semi-batch RO are compared with staged RO 629 without inter-stage pumps and/or ERDs, which might occur for BWRO when capital costs are 630 considered. In the BWRO case discussed earlier and shown in Fig. 6B (5840 ppm sodium 631 chloride feed, 90\% recovery, and circulation pressure loss of 0.1 bar), batch RO with ERDs is 632 equally promising as three-stage RO (with inter-stage pumps and ERDs), while semi-batch RO is 633 the least efficient process considered. If compared with staged RO without ERDs, batch RO with 634 ERDs hold greater promise, consuming 30\% less energy $\left(0.32 \mathrm{kWh} \mathrm{m}^{-3}\right)$ than two-stage RO 635 without ERDs and $21 \%$ less energy $\left(0.20 \mathrm{kWh} \mathrm{m}^{-3}\right)$ than three-stage RO without ERDs. In 636 contrast, semi-batch RO still consumes 14-29\% more energy than two-stage or three-stage RO 637 without ERDs. Semi-batch RO reduces energy consumption (38\%) only when compared to 638 staged RO without both inter-stage pumps and ERDs. FIGURE 6

641 Figure 6. Specific energy requirements calculated numerically through module-scale modeling for 642 conventional and alternative brackish water reverse osmosis (BWRO) processes. (A) Specific energy 643 versus typical system recoveries, $R$, for a circulation pressure loss, $\Delta$ Pcirc, of 0.1 bar. The gray shaded 644 area indicates the thermodynamic minimum specific energy required for desalination. (B) Excess specific 645 energy (i.e., specific energy, $S E$, minus the thermodynamic minimum specific energy, $S E m i n$ ) required 646 at a system recovery, $R$, of 0.9 for different circulation pressure losses. At this recovery, the minimum 
specific energy is $0.32 \mathrm{kWh} \mathrm{m}^{-3}$. Parameters used in modeling are listed in Table 1. Feed was modeled as

$6480.1 \mathrm{M}$ or $5840 \mathrm{ppm}$ sodium chloride. $\eta E R D$ : efficiency of energy recovery device.

649

650

651

652

653

654

\section{Other factors affecting implementation}

While energy usage is the main focus of this study, it is one of several important factors that influence the process design of RO plants. Other considerations include capital costs, process robustness and flexibility, and operational experience. Depending on the application, these factors can outweigh energy costs in decision-making. As such, we discuss these factors below to provide a more complete comparison of the process configurations.

\subsection{Capital costs}

Energy consumption translates to operational expenses; however, in many desalination applications, capital costs are of greater concern. It was for this reason that the two cases highlighted in Section $5-\mathrm{SWRO}$ at $50 \%$ recovery and high-salinity BWRO at $90 \%$ recovery - are relatively energy-intensive processes, since only for such situations could energy costs meaningfully affect design decisions. Even so, it is unclear whether the projected energy savings for these two cases would be significant enough to outweigh any potential increase in capital costs. Such an analysis would need to be made by plant designers.

While a thorough cost analysis is out of the scope of this study, the needed equipment in each process merits discussion. In $\mathrm{RO}$ with increased staging, the main additional equipment are the pumps, ERDs, piping, and pressure vessels needed for the added stage. In ideal batch RO, the pressurized, variable-volume feed tanks would likely be cost-prohibitive. Batch RO with ERDs should be more feasible, requiring modestly increased expenditure on ERDs due to the high levels of recycled flow. A more in-depth analysis would be required to assess whether increased capital costs in batch RO with ERDs would outweigh the energetic savings in BWRO found in this study, and how those costs compare with those of three-stage RO with inter-stage pumps and ERDs, a similarly efficient process.

Semi-batch RO on the other hand should not require considerably more equipment than onestage RO, and is likely the most favorable alternative process from a capital-cost perspective. For BWRO applications of semi-batch RO, the only notable additional equipment is the booster pump. However, as shown in this study, semi-batch RO is relatively inefficient in terms of energy usage for high-recovery BWRO, and would only save energy when compared with staged 
677 RO without inter-stage booster pumps. This increased energy consumption in semi-batch RO 678 could be important for high-TDS feeds, such as the one modeled in this study. For more typical 679 BWRO feeds of $\sim 1000$ ppm TDS, the increased energy consumption of semi-batch RO would be 680 relatively insignificant. For SWRO applications, semi-batch RO should again be competitive in 681 terms of capital costs. The number of pressure vessels is essentially doubled to allow storage of 682 fresh feed during a cycle (for energy recovery as described in Section 2), but with the benefit of 683 not requiring explicit ERDs. Since the simulated energetics in this study are similar to what has 684 already been reported [27, 29], semi-batch RO may be a cost-effective yet energy-saving process 685 design in SWRO desalination.

\subsection{Process robustness and flexibility}

Another important consideration is process robustness, for which batch and semi-batch RO processes likely have some advantages [37]. Most of the advantages stem from the independence of the per-pass and system recoveries, i.e., the ability to independently set target system recoveries and attain these target recoveries by simply changing the cycle time and feed pressure. This feature affords batch and semi-batch RO with increased process flexibility and contrasts with once-through RO, for which the physical design of the plant places constraints on system recovery. For example, batch and semi-batch RO processes should be more adaptable to varying feed conditions that impact water flux: if water flux is lower than expected due to high TDS or membrane fouling, the concentration cycle time can be extended to achieve the target recovery.

Batch and semi-batch RO may also be more robust in terms of fouling and scaling. The low per-pass recoveries result in a smaller decrease in water flux along the module compared with once-through RO, which should reduce the fouling risk in lead elements due to lower local fluxes [38]. Also, the cross-flow rate can be varied independently of system recovery in batch and semibatch RO, which may enable mitigation of fouling and scaling (without affecting recovery) through the use of high-cross-flow rinse steps during concentration cycles. In addition, because batch and semi-batch systems allow for variable hydraulic pressure, osmotic backflushing [39] could be readily incorporated when needed into batch and semi-batch cycles to remove foulants and scalants that have built up on the membrane surface. Lastly, it is possible that the cyclical nature of batch and semi-batch processes will help reduce the scaling of sparingly soluble salts (e.g., silica and calcium sulfate), potentially allowing for higher achievable recoveries than staged RO systems [40, 41]. Operating at high recoveries increases the likelihood that the 
concentrations of sparingly-soluble species will exceed their saturation levels. In batch and semibatch processes operating in such conditions, membranes would be exposed to super-saturated solutions for only a limited period of time at the end of a given cycle, after which the supersaturated solution is replaced with fresh (dilute) feed. In contrast, in staged RO the last elements would continually be exposed to super-saturated solutions.

\subsection{Operational experience}

714 The last consideration discussed here is the depth of operational experience for a given 715 process configuration, especially in the conservative water-treatment industry. Staged operations 716 are by far the most familiar "alternative" process, with decades of experience in plants around 717 the world. Semi-batch RO is also a proven technology, with several pilot and commercial 718 installations for industrial RO, BWRO, and SWRO operating since 2009 [37]. Most notably, these installations have shown that desalination membranes maintain performance despite the continually varying hydraulic pressures in semi-batch RO. The two batch RO processes, in contrast, have little to no operational experience. Employment of the batch RO with ERDs process should be straight-forward, since all of the required equipment are commonly used in desalination. The main question is whether efficiencies achieved by ERDs in typical one-stage SWRO can be achieved when operating with continually varying hydraulic pressure. As shown

725 in this study, ERD efficiencies upwards of $\sim 95 \%$ are generally needed for batch RO to reduce energy consumption. Employment of pressurized batch RO would hinge on the cost-effective and robust design of variable-volume pressure tanks.

\section{Conclusion}

In this study, we used analytical and numerical modeling to assess the energetics of timevariant (batch and semi-batch) processes and processes with increased staging. Through analytical modeling, we found that minimizing the circulation frictional pressure losses in the piping is relatively important in batch and semi-batch processes, and maximizing the ERD

734 efficiency is crucial for batch processes with ERDs, even more so than for conventional staged processes. Through numerical modeling, we found that batch-like processes and processes with increased staging would generally yield similar energetic reductions. For SWRO, semi-batch RO

737 and two-stage RO with inter-stage booster pump and ERDs are similarly promising for energy savings; the two process configurations are projected to save $13 \%$ and $15 \%$ energy, respectively, 
compared with one-stage RO with ERDs. For high-recovery BWRO with a relatively saline feed,

740 three-stage RO with inter-stage booster pumps and ERDs and batch RO with ERDs are the most

741 promising, projected to yield $14 \%$ and $15 \%$ energy savings, respectively, when compared with

742 two-stage RO with inter-stage pumps and ERDs. In contrast, semi-batch RO would save energy

743 only when compared with staged RO without inter-stage pumps and ERDs, the least efficient

744 manner of staged operation. Fully pressurized (ideal) batch RO was the most energy-efficient

745 configuration in both SWRO and high-recovery BWRO, but is likely an impractical solution due

746 to the need for variable-volume, high-pressure tanks.

747 Altogether, our results provide quantitative evidence that process design can result in 748 substantial energy savings compared with conventional processes, including for SWRO for 749 which energy consumption has largely plateaued. These findings are most relevant for 750 applications that produce a highly saline brine and therefore require large energy input, including 751 processes such as SWRO, high-recovery BWRO, and RO as part of a ZLD scheme. For lower 752 salinity applications, the factors discussed in Section 6 (capital costs, operational experience, and 753 process robustness and flexibility) will largely determine the choice of process configuration.

\section{Appendix A. Derivations of specific-energy approximations}

\section{A.1 One-stage RO}

Eq. 6 in the main text is an analytical approximation for the specific energy consumption in one-stage RO. Because one-stage RO is a continuous, non-transient process, the derivation is relatively straight-forward. We first start with the rate of energy consumption for the following 760 components:

Energy to match brine osmotic pressure:

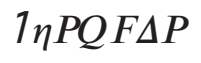

Recovered energy in energy recovery device:

$-1 \eta P \eta E R D Q F \Delta P(1-R)$

Frictional energy loss in piping: $\quad 1 \eta P Q F \Delta P$ circ

Frictional energy loss in membrane module:

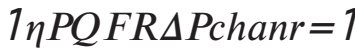

761 Combining the different terms and dividing by the permeate flow rate yields an equation for 762 specific energy consumption: 
$S E 1=1 \eta P Q P Q F \Delta P-\eta E R D Q F \Delta P(1-R)+Q F \Delta P c i r c+Q F R \Delta P c h a n r=1$

763 where $Q P$ is the permeate flow rate. Noting that $\Delta P=\pi O(1-R)$ and $R=Q P / Q F$, Eq. A.1 can be

764 simplified to give Eq. 6.

$S E 1 \pi O=1 \eta P 1 R 11-R-\eta E R D+\Delta$ pcirc $R+\Delta$ pchanr $=1$

765

766

767

Total energy to

match brine

osmotic pressure

in each stage:

Brine disposal

with energy

recovery:

Total frictional

energy loss in

$1 \eta P i=1 N Q F \Delta P \operatorname{Pirc} 1-r i-1$

piping:

Total frictional

energy loss in

membrane

$$
1 \eta P i=1 N Q F 1-r i-1 r \Delta P \operatorname{chanr}=1
$$

modules:

$$
1 \eta P i=1 N Q F \Delta P i 1-r i-1-Q F \Delta P i 1-r i=1 \eta P i=1 N Q F \Delta P i 1-r i-1 r
$$

$1 \eta P(1-\eta E R D) Q F \Delta P(1-R)$

768

769

Combining the different terms and dividing by the permeate flow rate yields an equation for 770 specific energy consumption:

$S E N=1 \eta P Q P i=1 N Q F 1-r i-1 r \Delta P i+Q F 1-r i-1 \Delta P c i r c+Q F 1-r i-1 r \Delta P c h a n r=1+1-\eta$

ERDQF $\triangle P 1-R$

771 Again, we note that $\Delta P=\pi O(1-R)$ and $R=Q P / Q F$. In addition, $\Delta P i=\pi O 1-r i$ and $1-r N=1-R$

772 or $r=1-1-R 1 / N$. Using these relations, Eq. A.2 can be simplified to: 
$S E N \pi O=1 \eta P 1 R N 1-R 1 N+1-N-\eta E R D+\Delta$ pcirc $1-1-R 1 / N+\Delta$ pchanr $=1$

773 In the main text, we leave the circulation pressure loss related to $r$ to highlight the relation

774 between $\Delta$ pcirc and the per-pass recovery. Plugging $r$ in yields Eq. 7.

$S E N \pi O=1 \eta P 1 R N 1-R 1 N+1-N-\eta E R D+\Delta p c i r c r+\Delta p c h a n r=1$

775

776

777

778

779

780

781

782

783

784

\section{A.3 Batch RO}

Eq. 8 in the main text is an analytical approximation for the specific energy consumption in batch RO. We only consider batch RO using energy recovery devices. To obtain the energetics of pressurized (ideal) batch RO, one can simply set $\eta E R D$ to 1 . We also assume batch RO operates with constant flux and varying pressure, allowing the permeate flow rate to be constant.

As for the above derivations, we first consider individual energetic contributions, which in this case are integrated with time, $t$, until the end of the batch cycle, $t \boldsymbol{B}$. Unlike for non-transient, staged processes, for batch RO we must consider volumes, not flow rates. Because the loop is depressurized before disposal, the small amount of energy required for brine disposal is neglected:

Total energy to match retentate osmotic pressure:

$1 \eta P O t B Q C \Delta P t d t$

Total energy recovered by energy recovery devices:

Total frictional energy loss in piping:

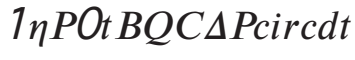

Total frictional energy loss in membrane modules:

\section{$1 \eta P O t B Q C r \Delta P c h a n r=1 d t$}

785 In these contributions, $Q C$ refers to the circulation flow rate, which corresponds to the flow 786 entering the membrane modules. The recycle flow rate is equal to $Q C 1-r$.

787 Combining the different terms and dividing by the permeate volume, $Q P t B$, yields an 788 equation for specific energy consumption:

$S E B=1 \eta P Q P t B O t B Q C \Delta P t d t-O t B \eta E R D Q C 1-r \Delta P t d t+O t B Q C \Delta P c i r c d t+O t B Q C r \Delta P c$ 
$h a n r=1 d t$

789 Only $\Delta P t$ is a function of $t$, mainly since it is a function of the osmotic pressure in the system, $\pi t$.

$790 \pi t$ is given by the following $\pi t=V T \pi O V T-Q P t=\pi 01-t \tau$, where $V T$ is the volume of the tank and

$791 \tau$ is the characteristic time of the system, with $\tau=V T Q P$. Paralleling the other configurations,

$792 \Delta P t=\pi t 1-r=\pi 01-t \tau 1-r$. In addition, we define $r=Q P Q C$ and $R=Q P t B V T=t B \tau$. Plugging

793 these terms into Eq. A.4 and simplifying, we get the following:

$S E B \pi O=1 \eta \operatorname{Prt} B O t B 11-r-\eta E R D 11-t \tau d t+\Delta p c i r c t B+\Delta p c h a n r=1 r t B$

794 Integrating the remaining integral yields the following:

$S E B \pi O=1 \eta \operatorname{Prt} B \tau 11-r-\eta E R D \ln 11-t \tau+\Delta$ pcirct $B+\Delta p c h a n r=1 r t B$

795 Simplifying further yields Eq. 8:

$S E B \pi O=1 \eta P 1 r R 11-r-\eta E R D \ln 11-R+\Delta p c i r c r+\Delta p c h a n r=1$

\section{A.4 Semi-batch RO}

Eq. 9 in the main text is an analytical approximation for the specific energy consumption in semi-batch RO. In semi-batch RO, the feed flow rate equals the permeate flow rate (i.e., $Q F=Q P) . Q C$ is again defined as the flow rate entering the membrane modules, with $Q C 1-r$ recycled and $r=Q P Q C$.

801 We again first consider individual energetic contributions, which are integrated with time, $t$, 802 until the end of the cycle, $t \boldsymbol{B}$. As for batch RO, we must consider volumes, not flow rates.

803 Because the loop is depressurized before disposal, brine disposal is neglected:

Total energy to match retentate osmotic pressure:

$$
1 \eta P O t B Q F \Delta P t d t=1 \eta P O t B Q P \Delta P t d t
$$

Total frictional energy loss in piping:

\section{$1 \eta P O t B Q C \Delta P c i r c d t$}

Total frictional energy loss in membrane modules:

804 Combining the different terms and dividing by the permeate volume, $Q P t B$, yields an 805 equation for specific energy consumption: 
$S E S B=1 \eta P Q P t B O t B Q P \Delta P t d t+0 t B Q C \Delta P c i r c d t+0 t B Q C r \Delta P c h a n r=1 d t$

806 In semi-batch RO, recovery differs from in batch RO, and is defined as the following:

$$
R=Q P t B Q P t B+V C=t B \tau 1+t B \tau,
$$

807

808

809

810

811

812

The hydraulic pressure is thus defined as:

$$
\Delta P t=\pi t 1-r=\pi 01+t \tau 1-r
$$

813 Plugging Eq. A.10 into Eq. A.7, integrating, and rearranging yields Eq. 9:

$S E S B \pi O=1 \eta P 2-R 21-r 1-R+\Delta p c i r c r+\Delta p c h a n r=1$

814

\section{Appendix B. Numerical module-scale analysis of reverse osmosis}

\section{B.1 Correlations used in module scale modeling}

A correlation based on equilibrium data from OLI Systems (Cedar Knolls, NJ) is used to model the dependence of osmotic pressure, $\pi$, on sodium chloride concentration, $c$ :

$\pi=44.2 c+4.57 c 2+0.553 c 3$

819 where the units of $\pi$ and $c$ are bar and $\mathrm{mol} \mathrm{L}^{-1}$, respectively. Eq. B. 1 is used to calculate $\pi F, m$ 820 and $\pi P$, which appear in Eq. 14 in the main text, from $c F, m$ and $c P$ respectively.

821 The feed-side mass transfer coefficient, $k F$, is calculated at each step using the following

822 Sherwood-Reynolds-Schmidt number correlation [33]:

$S h=0.16 \operatorname{Re} 0.605 \mathrm{Sc} 0.42$

$k F=S h D d h=0.16 \operatorname{Re} 0.605 S c 0.42 D d h$ 
823 where $\mathscr{D}$ is the diffusion coefficient of sodium chloride in water and $d h$ is the hydraulic diameter

824 of the membrane channel. A typical hydraulic diameter of $0.950 \mathrm{~mm}$ is assumed in this study

825 [35]. The dependence of $\mathscr{D}$ on solute concentration is given by the following correlation [36]:

$\mathscr{D}=6.73 \times 10-6 \exp 0.00903 c-8.43$

826 where the units of $\mathscr{D}$ and $c$ are $\mathrm{m}^{2} \mathrm{~s}^{-1}$ and $\mathrm{mol} \mathrm{L}^{-1}$, respectively. The temperature is assumed to be

827 constant at $298 \mathrm{~K}$ in Eq. B.4.

828 The frictional pressure loss in the feed-side membrane channel is calculated using an

829 established semi-empirical correlation [34, 35], given in Eq. 18 from the main text.

$d P \operatorname{chand} L=0.8 \operatorname{Re}-0.19 \operatorname{Re} 2 \rho v 2 d h 3 L$

830 where $d$ Pchan is the infinitesimal frictional pressure loss in the channel, $R e$ is the Reynolds number, $\rho$

831 is the fluid density, $v$ is the kinematic viscosity, $L$ is the length of the membrane channel, and $d h$

832 is the hydraulic diameter of the spacer-filled channel.

833 The fluid density is calculated using the following correlation [36]:

$\rho=460+212000+696 c$

834 where the units of $\rho$ and $c$ are $\mathrm{kg} \mathrm{m}^{-3}$ and $\mathrm{mol} \mathrm{L}^{-1}$, respectively. The temperature is assumed to be 835 constant at $298 \mathrm{~K}$ in Eq. B.5. The kinematic viscosity is calculated using a correlation for the 836 dynamic viscosity, $\mu$, divided by the fluid density calculated using Eq. B.5 [36].

$\mu=1.23 \times 10-6 \exp 0.124 c+6.59$

$v=\mu \rho$

837 where the units of $\mu$ and $c$ are $\mathrm{Pa}$ s and $\mathrm{mol} \mathrm{L}^{-1}$, respectively.

838 B.2 Numerical techniques used in module-scale modeling and specific-energy calculations

839 The numerical integrals in this study are evaluated using multistep RK4, a fourth-order Runge

840 Kutta method. The number of steps, $N$, is chosen such that doubling $N$ does not change the

841 results of the modeling by more than $0.01 \%$. The module-scale modeling presented in Section 5

842 is based on the numerical integration of Eqs. 12 and 13 from the main text. For these integrations, 
$843 N$ is typically between $10^{3}$ and $10^{4}$. For the time integrals that appear in Eqs. 22 and 23 from the

844 main text, $N$ is typically between 250 and 750 .

845

846

847

848

849

\section{B.3 Comparing the analytical and numerical optimal per-pass recovery}

The analytical optimal per-pass recovery for batch, $r B *$, and semi-batch, $r S B$ *, are given in Eqs. 10 and 11 from the main text, respectively. In Section 5.4 we use Eqs. 10 and 11, rather than a numerical minimization technique, to calculate $r B$ * and $r S B *$ respectively. Fig. B.1 illustrates how the specific energies of batch (red lines) and semi-batch (blue lines) vary with per-pass recovery, $r$, for SWRO (A) and BWRO (B). Our analytical values for $r B$ * and $r S B$ * are shown for comparison using dashed vertical lines. Fig. B.1 shows that our analytical approximations, Eqs. 10 and 11, are valid for SWRO and BWRO. Although the analytical $r B$ * and $r S B$ * values tend to underestimate the numerical optimal per-pass recovery, they do not lead to a significant overestimation of the minimum specific energy, $S E$, (the lowest point on each curve).

\section{FIGURE B.1}

Figure B.1 Specific energy requirements versus per-pass recovery, $r$, for batch and semi-batch reverse osmosis (RO). (A) Seawater RO (SWRO) case with inlet feed concentration 0.6 mol L$^{-1}$ sodium chloride and overall water recovery, $R$, of 0.5 . The optimal per-pass recoveries, $r *$, for batch and semi-batch RO were analytically determined using Eqs. 10 and 11 to be $r B *=0.13$ (dashed red line) and $r S B *=0.046$ (dashed blue line), respectively. (B) Brackish water RO (BWRO) case with inlet feed concentration of 0.1 mol L $\mathrm{L}^{-1}$ sodium chloride and $R=0.9$. The analytically determined optimal per-pass recoveries for batch and semi-batch RO are $r B *=0.14$ (dashed red line) and $r S B *=0.060$ (dashed blue line), respectively. Specific energies in (A) and (B) were calculated numerically through module-scale modeling. An energyrecovery-device efficiency, $\eta E R D$, of 0.98 was assumed for each case. Other parameters used in modeling are listed in Table 1.

\section{Acknowledgments}


We acknowledge the support received from the National Science Foundation through the Engineering Research Center for Nanotechnology-Enabled Water Treatment (ERC-1449500) and via Grant CBET 1437630. We also acknowledge the National Science Foundation Graduate Research Fellowship awarded to J.R.W.

\section{References}

876 1. M. Elimelech, The Global Challenge for Adequate and Safe Water, J. Water Supply Res.

2. M. Elimelech, W.A. Phillip, The Future of Seawater Desalination: Energy, Technology, and the Environment, Science 333 (2011) 712-717.

3. L.F. Greenlee, D.F. Lawler, B.D. Freeman, B. Marrot, P. Moulin, Reverse Osmosis Desalination: Water Sources, Technology, and Today's Challenges, Water Res. 43 (2009) 2317-2348.

4. C. Fritzmann, J. Lowenberg, T. Wintgens, T. Melin, State-of-the-Art of Reverse Osmosis Desalination, Desalination 216 (2007) 1-76.

5. National Research Council, Water Reuse: Potential for Expanding the Nation's Water Supply Through Reuse of Municipal Wastewater, National Academies Press, 2012.

6. G. Tchobanoglous, F.L. Burton, D.H. Stensel, Wastewater Engineering: Treatment and Reuse, 4th ed., McGraw-Hill, Boston, 2003.

7. M. Mickley, Survey of High-Recovery and Zero Liquid Discharge Technologies for Water Utilities, WateReuse Foundation, Alexandria, VA, 2008, pp. 143.

8. T. Tong, M. Elimelech, The Global Rise of Zero Liquid Discharge for Wastewater Management: Drivers, Technologies, and Future Directions, Environ. Sci. Tech. (2016). http://dx.doi.org/10.1021/acs.est.6b01000

9. J.R. Werber, A. Deshmukh, M. Elimelech, The Critical Need for Increased Selectivity, Not Increased Water Permeability, for Desalination Membranes, Environ. Sci. Tech. Lett. 3 (2016) 112-120.

10. L.F. Song, J.Y. Hu, S.L. Ong, W.J. Ng, M. Elimelech, M. Wilf, Performance Limitation of the Full-Scale Reverse Osmosis Process, J. Membr. Sci. 214 (2003) 239-244.

11. S. Lin, M. Elimelech, Staged Reverse Osmosis Operation: Configurations, Energy Efficiency, and Application Potential, Desalination 366 (2015) 9-14.

12. T. Qiu, P.A. Davies, Comparison of Configurations for High-Recovery Inland Desalination Systems, Water 4 (2012) 690-706.

13. C. Liu, K. Rainwater, L.F. Song, Energy Analysis and Efficiency Assessment of Reverse Osmosis Desalination Process, Desalination 276 (2011) 352-358.

14. R.L. Stover, Seawater Reverse Osmosis with Isobaric Energy Recovery Devices, Desalination 203 (2007) 168-175.

15. Flowserve Corporate Website, www.flowserve.com

16. C.R. Bartels, K. Andes, Consideration of Energy Savings in SWRO, Desal. Water Treat. 51 (2013) 717-725.

17. Energy Recovery Corporate Website, www.energyrecovery.com

18. J. Schwinge, P.R. Neal, D.E. Wiley, D.F. Fletcher, A.G. Fane, Spiral Wound Modules and Spacers, J. Membr. Sci. 242 (2004) 129-153. 
19. A. Drak, M. Adato, Energy Recovery Consideration in Brackish Water Desalination, Desalination 339 (2014) 34-39.

20. R.I. Bratt, Method and Apparatus for Fluid Treatment by Reverse Osmosis, U.S. Patent 4,814,086, 1989.

21. P.A. Davies, A Solar-Powered Reverse Osmosis System for High Recovery of Freshwater from Saline Groundwater, Desalination 271 (2011) 72-79.

22. E. Oklejas Jr., Batch-Operated Reverse Osmosis System with Manual Energization, U.S. Patent 7,892,429 B2, 2011.

23. E. Oklejas Jr., Continuous Process Batch-Operated Reverse Osmosis System with In-Tank Membranes and Circulation, U.S. Patent 8,377,302 B2, 2013.

24. S. Mirza, Reduction of Energy Consumption in Process Plants Using Nanofiltration and Reverse Osmosis, Desalination 224 (2008) 132-142.

25. A. Tarquin, M. Fahy, J. Balliew, Concentrate Volume Reduction Research in El Paso, Texas, World Environmental and Water Resources Congress, 2010, pp. 3507-3518.

26. B. Sauvet-Goichon, Ashkelon Desalination Plant - A Successful Challenge, Desalination 203 (2007) 75-81.

27. A. Efraty, R.N. Barak, Z. Gal, Closed Circuit Desalination - A New Low Energy High Recovery Technology Without Energy Recovery, Desal. Water Treat. 31 (2012) 95-101.

28. L. Szucz, A. Szucs, Method and Apparatus for Treating Fluids Containing Foreing Materials by Membrane Filter Equipment, U.S. Patent 4,983,301, 1991.

29. Z. Gal, A. Efraty, CCD Series no. 18: Record Low Energy in Closed-Circuit Desalination of Ocean Seawater with NanoH2O Elements Without ERD, Desal. Water Treat. 57 (2015) 9180-9189.

30. A.Z. Zhu, P.D. Christofides, Y. Cohen, Effect of Thermodynamic Restriction on Energy Cost Optimization of RO Membrane Water Desalination, Ind. Eng. Chem. Res. 48 (2009) 6010-6021.

31. R.W. Baker, Membrane Technology and Applications, John Wiley \& Sons, Ltd, 2012.

32. G.M. Geise, D.R. Paul, B.D. Freeman, Fundamental Water and Salt Transport Properties of Polymeric Materials, Prog. Polym. Sci. 39 (2014) 1-42.

33. C.P. Koutsou, S.G. Yiantsios, A.J. Karabelas, A numerical and experimental study of mass transfer in spacer-filled channels: Effects of spacer geometrical characteristics and Schmidt number, J. Membr. Sci. 326 (2009) 234-251.

34. C.P. Koutsou, S.G. Yiantsios, A.J. Karabelas, Direct numerical simulation of flow in spacer-filled channels: Effect of spacer geometrical characteristics, J. Membr. Sci. 291 (2007) 53-69.

35. G. Schock, A. Miquel, Mass transfer and pressure loss in spiral wound modules, Desalination 64 (1987) 339-352.

36. M. Taniguchi, M. Kurihara, S. Kimura, Behavior of a reverse osmosis plant adopting a brine conversion two-stage process and its computer simulation, J. Membr. Sci. 183 (2001) 249-257.

37. R.L. Stover, New High Recovery Reverse Osmosis Water Treatment for Industrial, Agricultural, and Municipal Applications, IDA World Congress, International Desalination Association, Tianjin, China, 2013.

38. E.M.V. Hoek, A.S. Kim, M. Elimelech, Influence of Crossflow Membrane Filter Geometry and Shear Rate on Colloidal Fouling in Reverse Osmosis and Nanofiltration Separations, Environ. Eng. Sci. 19 (2002) 357-372. 
959 39. E. Bar-Zeev, M. Elimelech, Reverse Osmosis Biofilm Dispersal by Osmotic Back-Flushing: 960 Cleaning via Substratum Perforation, Environ. Sci. Technol. Lett. 1 (2014) 162-166.

961 40. Z. Gal, J. Septon, A. Efraty, A.-M. Lee, CCD Series no-20: High-Flux Low-Energy

$962 \quad$ Upgrade of Municipal Water Supplies with 96\% Recovery for Boiler-Feed and Related 963 Applications, Desal. Water Treat. (2016) 1-9.

964 41. R.Y. Ning, A.J. Tarquin, J.E. Balliew, Seawater RO Treatment of RO Concentrate to 965 Extreme Silica Concentrations, Desalin. Water Treat. 22 (2010) 286-291. 